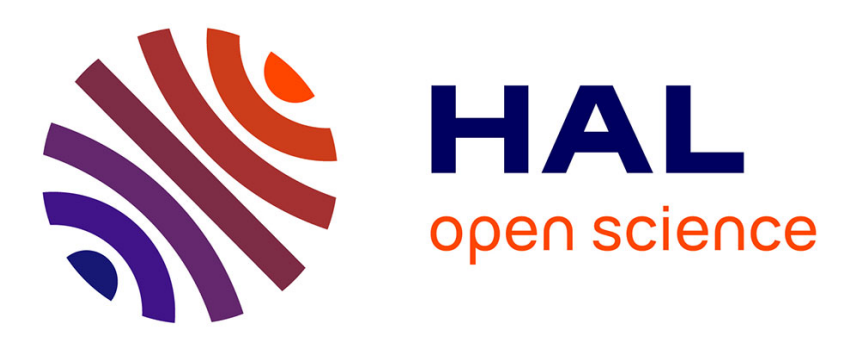

\title{
Influence of the magnetic state on the voltage-controlled magnetoelectric effect in a multiferroic artificial heterostructure YIG/PMN-PZT
}

Jianyun Lian, Freddy Ponchel, Nicolas Tiercelin, Liuyang Han, Ying Chen, Denis Remiens, Tuami Lasri, Genshui Wang, Philippe Pernod, Wenbin Zhang, et al.

\section{To cite this version:}

Jianyun Lian, Freddy Ponchel, Nicolas Tiercelin, Liuyang Han, Ying Chen, et al.. Influence of the magnetic state on the voltage-controlled magnetoelectric effect in a multiferroic artificial heterostructure YIG/PMN-PZT. Journal of Applied Physics, 2018, 124 (6), pp.064101. 10.1063/1.5037057 . hal-02127944

\section{HAL Id: hal-02127944 \\ https://hal.science/hal-02127944}

Submitted on 12 Oct 2020

HAL is a multi-disciplinary open access archive for the deposit and dissemination of scientific research documents, whether they are published or not. The documents may come from teaching and research institutions in France or abroad, or from public or private research centers.
L'archive ouverte pluridisciplinaire HAL, est destinée au dépôt et à la diffusion de documents scientifiques de niveau recherche, publiés ou non, émanant des établissements d'enseignement et de recherche français ou étrangers, des laboratoires publics ou privés. 


\title{
Influence of the magnetic state on the voltage-controlled magnetoelectric effect in a multiferroic artificial heterostructure YIG/PMN-PZT
}

\author{
Jianyun Lian, ${ }^{1,2,3}$ Freddy Ponchel, ${ }^{2}$ Nicolas Tiercelin, ${ }^{2}$ Liuyang Han, ${ }^{1,2,3}$ Ying Chen, ${ }^{1,3, a)}$ \\ Denis Rémiens, ${ }^{2, b)}$ Tuami Lasri, ${ }^{2}$ Genshui Wang, ${ }^{1}$ Philippe Pernod, ${ }^{2}$ Wenbin Zhang, ${ }^{1}$ \\ and Xianlin Dong ${ }^{1, c)}$ \\ ${ }^{1}$ Key Laboratory of Inorganic Functional Materials and Devices, Shanghai Institute of Ceramics, \\ Chinese Academy of Sciences, 1295 Dingxi Road, Shanghai 200050, People's Republic of China \\ ${ }^{2}$ Université Lille, CNRS, Centrale Lille, ISEN, Université Valenciennes, UMR 8520-IEMN, LIA LICS/LEMAC, \\ F-59000 Lille, France \\ ${ }^{3}$ University of Chinese Academy of Sciences, 19 A Yuquan Rd., Shijingshan District, Beijing 100049, \\ People's Republic of China
}

(Received 20 April 2018; accepted 21 July 2018; published online 8 August 2018)

\begin{abstract}
The artificial multiferroic heterostructure used in this work is composed by an yttrium iron garnet (YIG) film deposited by radio frequency (rf) magnetron sputtering on a metalized PMN-PZT ceramic. We demonstrate, thanks to the well-known converse magnetoelectric (CME) coupling, the control of the magnetic state of the YIG film by means of a low electric field applied to the PMNPZT ceramic. In particular, it is shown that the variations of the magnetization induced by the strain are functions of the magnetic sate of the film. It is shown that the maximum amplitude variation is observed at the coercive magnetic field $(H c)$, whereas when $H$ increases, the strain effect has a limited impact on the film magnetization. A second effect has also been remarked on the magnetization of the YIG film but only after the first strain induced cycle has been applied. These variations, observed only under low applied magnetic fields, can be attributed to the re-orientation of some magnetic moments which are easy to switch under low magnetic fields. We find that after poling the YIG film, i.e., applying a magnetic field on it, the initial state is restored. The CME coupling coefficient is determined for different magnetic states of the YIG film, and the maximum value $11 \times 10^{-8} \mathrm{~s} / \mathrm{m}$ is obtained at $H=H c$ and $E=E c$ (the coercive field of the PMN-PZT ceramic). The relative susceptibility tunability available for the heterostructure proposed is $16.8 \%$ for an electric field applied between 0 and $-4 \mathrm{kV} / \mathrm{cm}$. Published by AIP Publishing.

https://doi.org/10.1063/1.5037057
\end{abstract}

\section{INTRODUCTION}

Recently, the magnetoelectric (ME) effect which results in a dielectric polarization variation of a material under a magnetic field or a magnetization variation under an electric field has become the focus of significant fundamental research interest and an exciting new frontier due to its wide range of potential applications. ME materials or composite structures have found applications in different fields such as power harvesting, current transformers, magnetic field sensors, current sensors, information storage (ME random access memory: MERAM), and novel electrostatically tunable microwave magnetic devices for telecommunication systems such as filters, resonators, inductors, and phase shifters. ${ }^{1-8}$

In naturally occurring multiferroics, ME coupling is often weak, and consequently, new classes of artificially structured composite materials that combine dissimilar magnetic and ferroelectric systems are being developed to optimize order parameter coupling. ${ }^{9,10}$ An alternative to engineering enhanced ME effects is to introduce indirect coupling, via strain, between two materials such as a ferromagnetic and a ferroelectric material. The existence of a

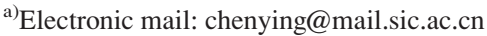

b) Electronic mail: denis.remiens@univ-valenciennes.fr

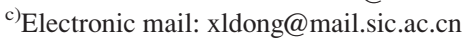

strain coupling requires intimate contact between a magnetostrictive (or piezomagnetic) material and a piezoelectric (or electrostrictive) material. Each phase may then be independently optimized, and the coupling performance is investigated afterward. This enables the control of both the dielectric polarization $P$ by a magnetic field $H$ [direct magnetoelectric effect (DME): $d P=\alpha_{E} d H$, with $\alpha_{E}$ being the ME coupling coefficient] and the manipulation of magnetization $M$ by an electric field $E$ [converse magnetoelectric effect (CME): $\mu_{0} d M=\alpha_{H} d E$, with $\alpha_{H}$ being the ME coupling coefficient and $\mu_{0}$ the vacuum permeability]. The ME efficiency, at room temperature, is quantified in terms of the ME coupling coefficient $\left(\alpha_{E}\right.$ or $\left.\alpha_{H}\right)$, which represents the coupling efficiency between the electric and magnetic systems. This is considered as the figure of merit for the ME coupling strength. ${ }^{11,12}$

Many studies have been conducted on the fundamental understanding, fabrication processes, and applications of ME artificial composite systems in the last four decades, which has brought the technology closer to the realization of practical devices. The possibility of using an electric field to control the magnetization of a ferromagnetic material is a fundamental topic, and recently, there has been significant effort to improve the controllability. The CME effect opens a large range of opportunities for the electric control of $M$, as 


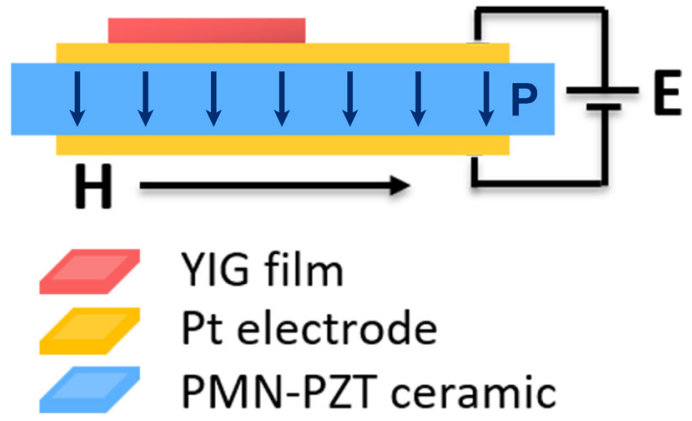

FIG. 1. The schematic of the YIG/Pt/PMN-PZT/Pt heterostructure.

shown in recent studies on voltage-controlled magnetic anisotropy in appropriately synthesized FM/FE heterostructures. ${ }^{13,14}$ The FE materials usually used are PZT ceramics and PMN-PT single crystals since they have a high $d_{33}$ coefficient and a large field-induced strain, while the FM materials used are typically metallic magnetic materials thanks to their excellent magnetostrictive properties. Other studied systems also include spinel ferrites or hexagonal ferrites for the magnetic phase. Briefly speaking, the deformation of the piezoelectric layer caused by the applied electric field is transferred to the mechanically coupled ferrite layer so that the elastic stress in the ferrite manifests a first-order uniaxial magneto-crystalline anisotropy and produces a change in the magnetization. ${ }^{15-17}$

Ferrites are an important class of materials for use in microwave devices such as resonators, filters, and phase shifters. ${ }^{18,19}$ Low-loss ferromagnets including yttrium iron garnet (YIG), lithium ferrite, and hexagonal ferrites are used in devices operating in the frequency band of $1-70 \mathrm{GHz}$. In particular, the magnetic properties of YIG have been largely studied for microwave devices. Indeed, recently, microwave YIG isolators and circulators have been investigated for wireless communication systems. ${ }^{20-23}$

As the applications envisaged within the framework of this work are tunable structures at high frequency, YIG is selected as the ferromagnetic material. The fabricated heterostructure is therefore composed of a YIG thin film $(200 \mathrm{~nm}$ thick) deposited by rf magnetron sputtering on a piezoelectric ceramic $(0.5 \mathrm{~mm}$ thick). The piezoelectric material is lead magnesium niobate-lead zirconate titanate (PMN-PZT) with a composition near the morphotropic phase boundaries: 0.25 PMN-0.75 PZT. With this composition, we have demonstrated the excellent properties of this material: low coercive field, strong $d_{33}$, and strain. The voltage tuning of magnetization $M(V)$ is performed by an homemade instrument which combines a Vibration Sample Magnetometer (VSM) and an external high voltage source $(0- \pm 15 \mathrm{kV})$ via two copper wires attached to the upper and lower electrodes of the sample in the VSM test chamber. The instrument allows polarizing the sample by applying a high DC voltage to the ceramic and modifying the magnetic state of YIG under a magnetic field simultaneously.

\section{EXPERIMENTS}

A description of the sample is shown in Fig. 1, and the ceramic used as a substrate is chosen, among the different kinds of ceramics available, according to its piezoelectric performance. In our case, we have decided to work with a PMN-PZT ceramic $(8 \mathrm{~mm} \times 8 \mathrm{~mm}-0.5 \mathrm{~mm}$ in thickness $)$. Recently, we have demonstrated ${ }^{24}$ that at the morphotropic phase boundary, a sample $(0.25$ PMN-0.75 PZT) can reach the maximum strain $(2.5 \%)$ at a small coercive electric field $(E c=4.43 \mathrm{kV} / \mathrm{cm})$ with an AC electric field of $15 \mathrm{kV} / \mathrm{cm}$ and a frequency of $0.01 \mathrm{~Hz}$ (Fig. 2). We observe a variation of $E c$ with the working frequency, i.e., a decrease in $E c$ when $f$ decreases, and this behavior is classical. The PMNPZT ceramic can reach saturation at a very low electric field and its maximum strain value occurs at the coercive field, which are of great interest of this material.

Before the film deposition on the ceramic, a very important step is the polishing process. The face/interface quality between the ceramic and the films can strongly affect the strain mediated converse magnetoelectric coupling. ${ }^{25}$ Figure 3(a) shows a typical AFM image of the ceramic surface after polishing; the surface roughness is estimated to be $10 \mathrm{~nm}$ (mean value). With our polishing system, it is quite difficult to obtain a better quality, but such a level of roughness allows however envisaging the growth of films. In the first step, Pt electrodes are deposited on both sides of the substrate, and these electrodes are needed to apply an electric
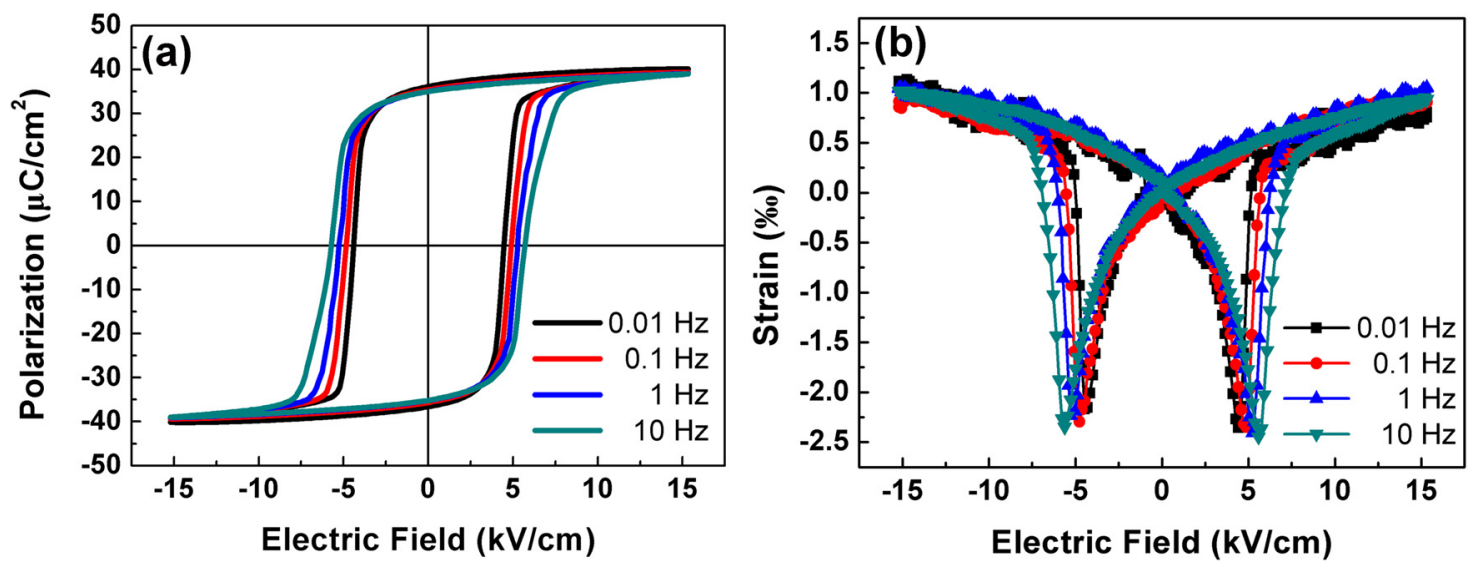

FIG. 2. The hysteresis loops of polarization vs. E-field (a) and field-induced strain curves (b) of a 0.5 mm-thick PMN-PZT ceramic with different working frequencies $(0.01,0.1,1$, and $10 \mathrm{~Hz})$. 

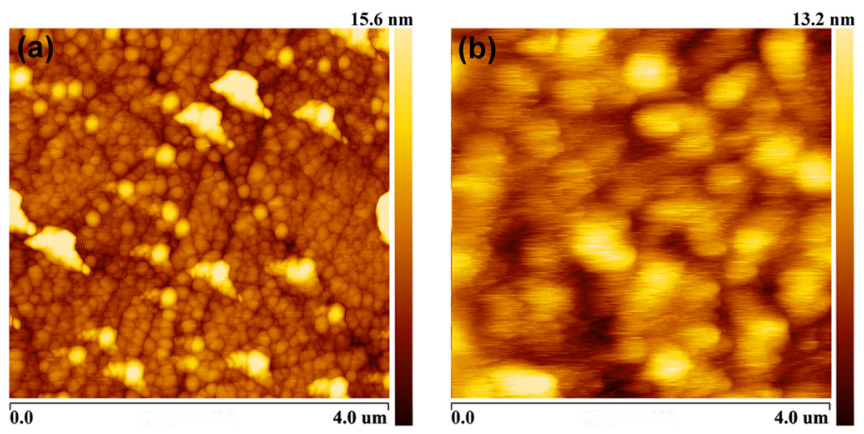

FIG. 3. The AFM images of a PMN-PZT ceramic after polishing (a) and the YIG film grown on the ceramic (b).

TABLE I. The preparing conditions of the YIG thin film.

\begin{tabular}{lc}
\hline \hline Parameters & Values \\
\hline Base pressure $(\mathrm{Pa})$ & $3.0 \times 10^{-4}$ \\
Substrate temperature $\left({ }^{\circ} \mathrm{C}\right)$ & $\mathrm{RT}$ \\
Sputtering atmosphere & $\mathrm{Ar}$ \\
Sputtering pressure $(\mathrm{Pa})$ & 0.55 \\
Sputtering power $(\mathrm{W})$ & 100 \\
Sputtering time $(\mathrm{h})$ & 5 \\
Annealing temperature $\left({ }^{\circ} \mathrm{C}\right)$ & 750 \\
Holding time $(\mathrm{h})$ & 1 \\
\hline \hline
\end{tabular}

field on the ceramic. A metallic mask is used to define the zone of deposition (Fig. 1). The YIG thin film, $200 \mathrm{~nm}$ thick, is then deposited by $\mathrm{rf}$ magnetron sputtering also through this type of mask. The sputtering and post-annealing conditions are given in Table I; they are the same as for deposition on silicon except the annealing treatment. ${ }^{26}$ It is made at the same temperature $\left(750^{\circ} \mathrm{C}\right)$ but with a shorter duration $(1 \mathrm{~h})$ to avoid (or limit) the outflow of $\mathrm{Pb}$ in PMN-PZT at high temperature. We have also analyzed the YIG film surface roughness by AFM [Fig. 3(b)] and the microstructure by SEM [Figs. 4(a) and 4(b)]. Typically, the roughness is on the order of $7 \mathrm{~nm}$ (mean value), and the structure is dense with very fine and uniform grains. The interface is very clear, indicating no significant diffusion between the film and electrode.

\section{RESULTS AND DISCUSSION}

Figure 5 presents the X-ray diffraction pattern of the YIG film deposited on the Pt/PMN-PZT/Pt substrate. As we can see although the diffraction peaks of the YIG film are much weaker than the diffraction peaks of the substrate and electrodes, all of them correspond to the pure cubic $\mathrm{Y}_{3} \mathrm{Fe}_{5} \mathrm{O}_{12}$ phase and there is no second phase.

To finalize the preparation of the sample for future characterization studies, electrical connections must be available on both sides of the substrate on the Pt layers. However, since it is necessary to apply high voltage on the ceramic, some protections must be taken for the electrical connections. To that end, the connections on the sample holder are electrically isolated from the measuring equipment, preventing possible damage in the VSM. After the preparation of the sample and the fabrication of the sample holder, the evaluation of the sample properties can be achieved, which is presented thereafter.

The first measurements concern the evaluation of the magnetic properties of the YIG film deposited on the Pt/ PMN-PZT/Pt substrate. The VSM used for both $M(H)$ and $M(E)$ characterization studies is an ADE model EV9. Its electromagnet can operate up to $2.1 \mathrm{~T}$, the resolution is on the order of the micro-emu, and the frequency of vibration is fixed to $75 \mathrm{~Hz}$. As our system allows polarizing the sample under test by an electric field, it is thus possible to simultaneously apply a DC high voltage to the ceramic and modify the magnetic state of the YIG film by applying an in-plane magnetic field.

In order to obtain the $M(H)$ loops of the YIG film only, i.e., without the presence of the ceramic, the electrodes, and the wires, it is necessary to implement a calibration step beforehand. So, we have recorded the magnetic response of the ceramic with and without electrodes and the wire connection. Finally, the effect of these external elements is taken into account by means of a de-embedding procedure to extract only the properties of the film. The $M(H)$ magnetic loops are presented in Fig. 6. The related saturation magnetization $(M s)$, remnant magnetization $(M r)$, coercive field $(H c)$, and magnetic susceptibility $(\chi)$ are summarized in Table II. We note some variations of these main characteristics with regard to a YIG film of the same thickness deposited on $\mathrm{SiO}_{2} / \mathrm{Si}^{26}$ The film roughness, lattice structure, defects, and the interface quality can explain these dissimilarities.

As the magnetic properties of the YIG film are perfectly known now, the next step of this study is to examine the variations of these properties according to an electric field applied on the ceramic. As said before, our VSM system
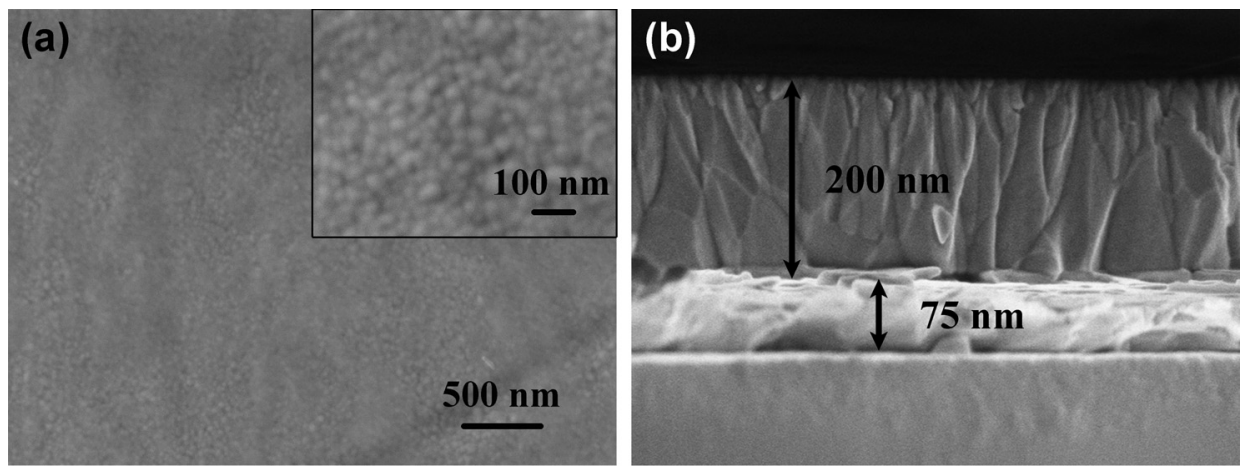

FIG. 4. The surface (a) and crosssectional SEM images (b) of the YIG film grown on the PMN-PZT ceramic. 


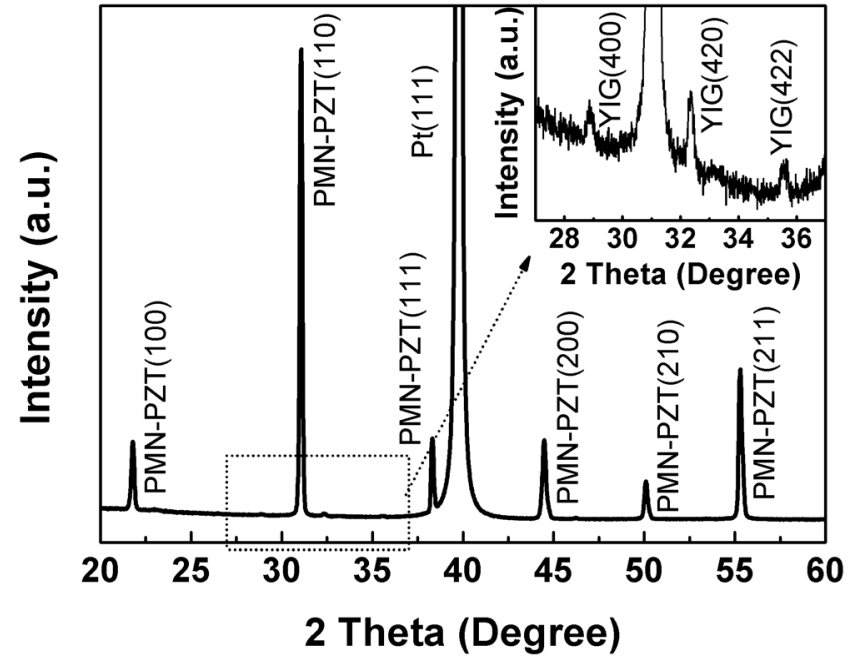

FIG. 5. The X-ray diffraction pattern of the YIG/Pt/PMN-PZT/Pt heterostructure. The inset shows an enlarged region containing the main peaks of the YIG film.

offers the possibility to apply a DC voltage on the ceramic when the YIG film is in different magnetic states (as a function of $H)$. So, we have the opportunity to measure the $M(E)$ response of YIG under different applied $H$.

In this study, the YIG film is submitted to various magnetic fields going from 0 to $320 \mathrm{Oe}$, leading to different magnetization states. In particular, $M(E)$ loops are measured for seven different magnetic fields $(320,160,80,40,20,10$, and 0 Oe). First, we start with a field of 320 Oe [Fig. 7(a)], the film is in a saturated state, and we decrease the magnetic field (passing by $H c$ ) to arrive to 0 Oe. Under a specific magnetic field, the cycle for the change of the electric field is as follows: $0 \mathrm{kV} / \mathrm{cm},-15 \mathrm{kV} / \mathrm{cm}, 15 \mathrm{kV} / \mathrm{cm},-15 \mathrm{kV} / \mathrm{cm}$, and stops at $0 \mathrm{kV} / \mathrm{cm}$. We note in Fig. 7 that all the $M(E)$ curves follow the butterfly loop as it is observed for the strain in the PMN-PZT ceramic under an electric field (Fig. 2). This behavior is observed regardless of the magnetic state of the film.

These results demonstrate clearly that the magnetization variation is induced by the converse magnetoelectric effect

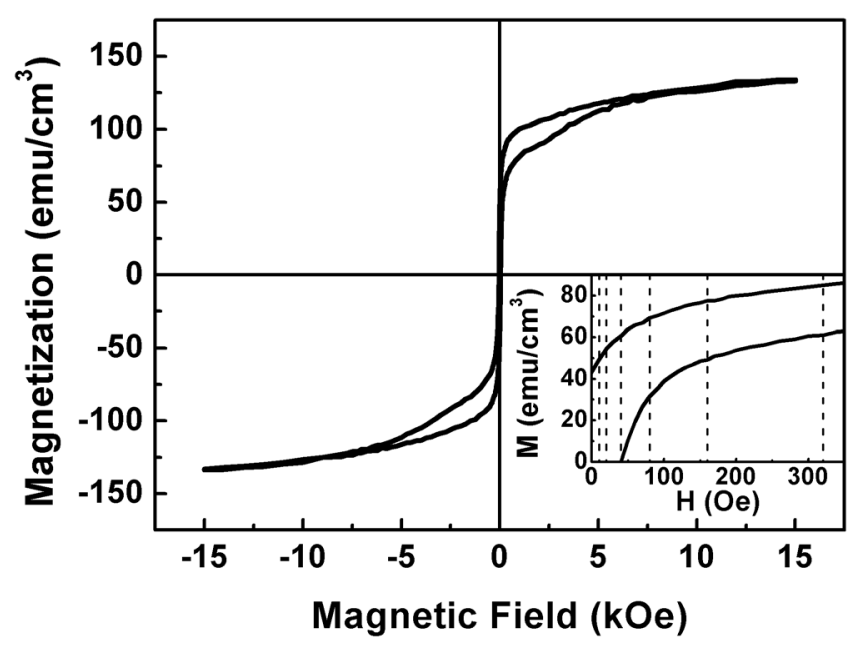

FIG. 6. The magnetic hysteresis loop and its enlarged curve (inset) of the YIG film excluding the effect of Pt/PMN-PZT/Pt and wires.
TABLE II. The corresponding saturation magnetization $(M s)$, remnant magnetization $(\mathrm{Mr})$, coercive field $(H c)$, and magnetic susceptibility $(\chi)$ of YIG thin films grown on Pt/PMN-PZT/Pt.

\begin{tabular}{lccr}
\hline \hline$M s\left(\mathrm{emu} / \mathrm{cm}^{3}\right)$ & $M r\left(\mathrm{emu} / \mathrm{cm}^{3}\right)$ & $H c(\mathrm{Oe})$ & $\chi$ \\
\hline 133.8 & 43.5 & 40.1 & 15.9 \\
\hline \hline
\end{tabular}

on the heterostructure made of alternating materials of ferroelectric and ferromagnetic phases. In fact, the electro-strain originated from the ferroelectric material generates a modification of the magnetization state of the ferromagnetic material. We can define the performance of our structure by the magnetization variations under the effect of the applied electric field and related to the magnetic state of the material. Figure 8 shows the variation of $\Delta M / M_{O i}$ as a function of the bias $\mathrm{H}$ field, where $\Delta M$ is the difference of $M$ at $E_{C}$ in the ascending and descending parts of the E field cycle, indicating the maximum $M$ variation in the $M(E)$ curve originated from the maximum strain effect near $E c$; $M_{O i}$ is the film initial magnetization state at $0 \mathrm{kV} / \mathrm{cm}$ (Fig. 7). It is noticed that $\Delta M / M_{0 i}$ first increases between $H=0$ and $H=H c$ (highest value for $\Delta M / M_{O i}$ ) and then it decreases. It is thus confirmed that the electric field applied to the ceramic is the most effective when the film is in this magnetic state $(H=H c)$. One can also note (Fig. 8) that even without $H$, a variation exists $\left(\Delta M / M_{O i}=6.5 \%\right)$, and this result is very important for future applications. In contrast, under a high magnetic field, the mechanical strain effect has a limited impact on the film magnetization.

A second effect appears which induces also a change in the magnetization. Actually, a difference of the magnetization between the initial state $\left(M_{O i}\right)$, at the beginning of the experiment, and the magnetization state at the end of the test $\left(M_{O f}\right)$ is observed. $M_{O i}$ is of course in relation with $H$ applied to the YIG film just before the measurement. For example, in Fig. 7(a) $(H=320 \mathrm{Oe}), M_{O i}$ associated is $86.5 \mathrm{emu} / \mathrm{cm}^{3}$ (confirmed by Fig. 6). In the same way, in Fig. $7(\mathrm{~g})(H=0 \mathrm{Oe})$, $M_{O i}=42.5 \mathrm{emu} / \mathrm{cm}^{3}$; this value is very close to $M r$. The ratio $\left(M_{O i}-M_{O f}\right) / M_{O i}$ (Fig. 7) depends on the magnetic state of the YIG film. This ratio, as shown in Fig. 9, gets its maximum at 0 Oe and then decreases, as a function of $H$, down to zero for $H \geq 150$ Oe.

To analyze finely the factors that cause the variation of $M_{O i}$ and $M_{O f}$, we have repeated 4 times the experiments. An example is presented in Fig. 10(a) for a saturated film $(H=3000 \mathrm{e})$ and Fig. 10(b) for a YIG film slightly magnetized $(H=30 \mathrm{Oe})$. The YIG film is "saturated" only before the measurement of the first curve, which means that the initial state for the second cycle is the one obtained at the end of the first cycle $\left(M_{O i 2}=M_{O f 1}\right)$.

Different observations can be done from these figures. When the magnetic material is strongly magnetized [Fig. 10(a)], the states of $M_{O i}$ and $M_{O f}$ are practically the same, whereas a difference between these two states is noticed when a low magnetic field is applied [Fig. 10(b)]. This difference $\left(M_{O i 1}-M_{O f 1}\right)$, on the order of $2 \mathrm{emu} / \mathrm{cm}^{3}$ in this example, appears only after the strain induced first cycle, which could be explained by the fact that after undergoing 

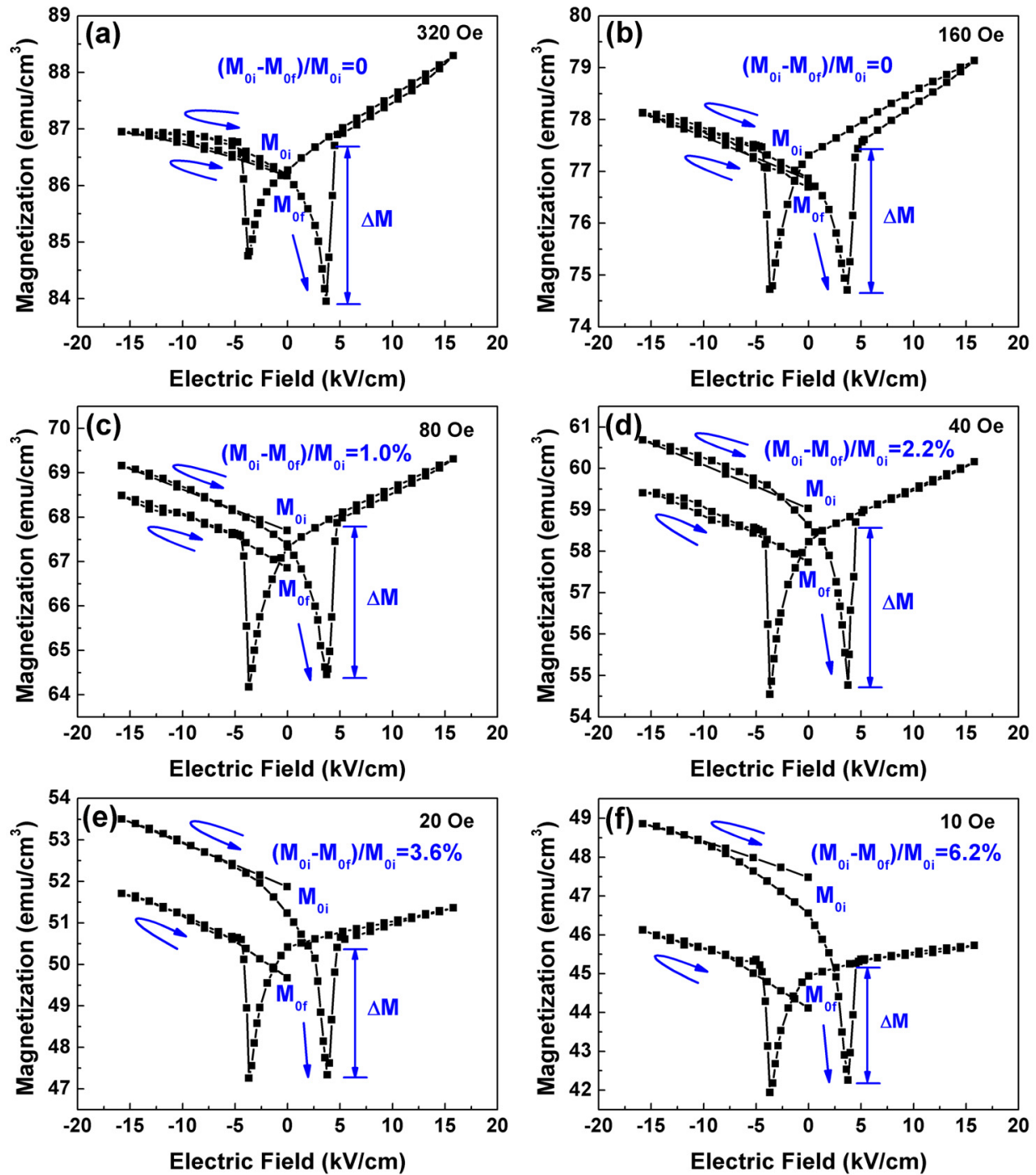

FIG. 7. The electric field dependency of the magnetization of the YIG film without the effect of Pt/PMN-PZT/Pt and wires under a series of magnetic fields $(320,160,80,40,20,10$, and $0 \mathrm{Oe})$. The trend of all the curves can be illustrated by the arrows. $\Delta M$ is the difference of $M$ at $E_{C}$ in the ascending and descending parts of the $\mathrm{E}$ field cycle. $M_{O i}$ and $M_{O f}$ are the initial and the final magnetization, respectively, at $0 \mathrm{kV} / \mathrm{cm}$.

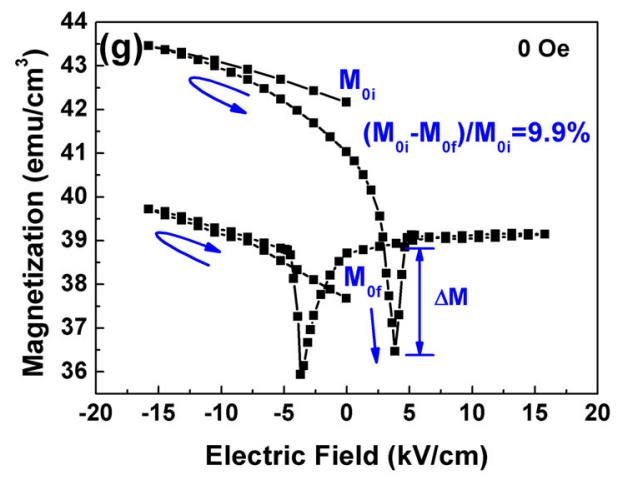

the strain effect, some magnetic moments do not return to their initial state under low magnetic fields. However, in the repeated cases, the final magnetization has been found to be equal to the initial one $\left(M_{O f j}=M_{O i j}, j=2,3\right.$, and 4). It is thus clear that only after the first cycle, the sample experienced that some magnetic moments are switched. In the subsequent cycles, the moments keep their position, and consequently, the magnetization remains constant. Under high magnetic fields, this phenomenon is not observed [Fig. 10(a), repeated also 4 times] certainly because, as for the strain effect, under a strong field, the magnetic moments are solidly aligned and it is difficult to switch them.
It has also been observed that the magnetization lost $\left(M_{O i}-M_{O f}\right)$ after the first cycle (cycle 1) can be recovered by re-saturating the YIG film, i.e., applying a magnetic field on it. As an illustration, we present in Fig. 11 an example where we have chosen $-15 \mathrm{kV} / \mathrm{cm}$ as the initial $\left(M_{i}\right)$ and final $\left(M_{f}\right)$ points of the cycles (for the 3 cycles: $-15 \mathrm{kV} / \mathrm{cm}, 0 \mathrm{kV} / \mathrm{cm}$, $15 \mathrm{kV} / \mathrm{cm}, 0 \mathrm{kV} / \mathrm{cm}$, and $-15 \mathrm{kV} / \mathrm{cm})$. The measurements are made at $H=0$ Oe where we have observed the maximum of variation. We retrieve the same phenomena as before; in this case, $M_{i 1}-M_{f 1}$ is larger $\left(4 \mathrm{emu} / \mathrm{cm}^{3}\right)$ in agreement with the $M(H)$ curve, and for the second curve, $M_{f 2}=M_{i 2}$. Before the third cycle, we poled the YIG film with a magnetic field of 


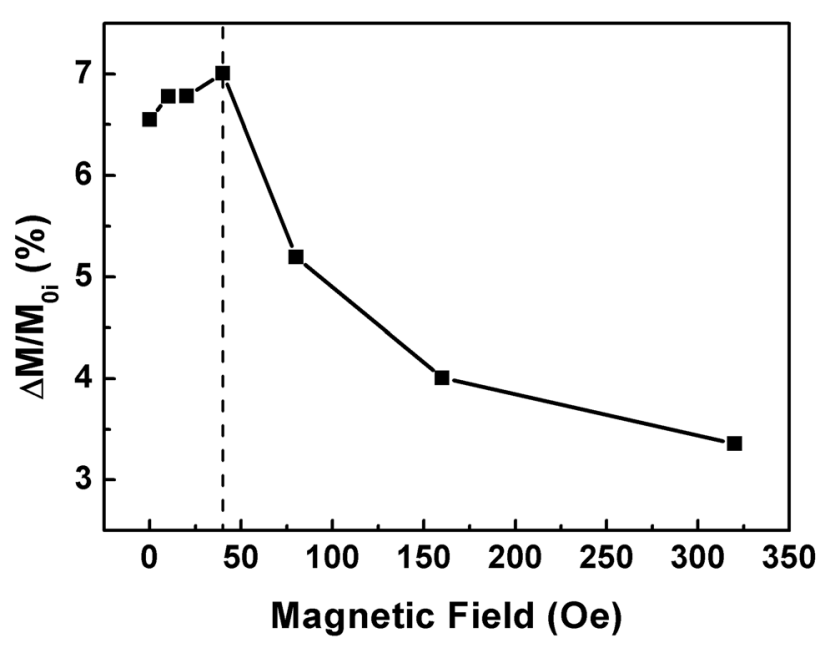

FIG. 8 . The magnetization variations $\left(\Delta M / M_{O i}\right)$ induced by strain as a function of the applied magnetic field.

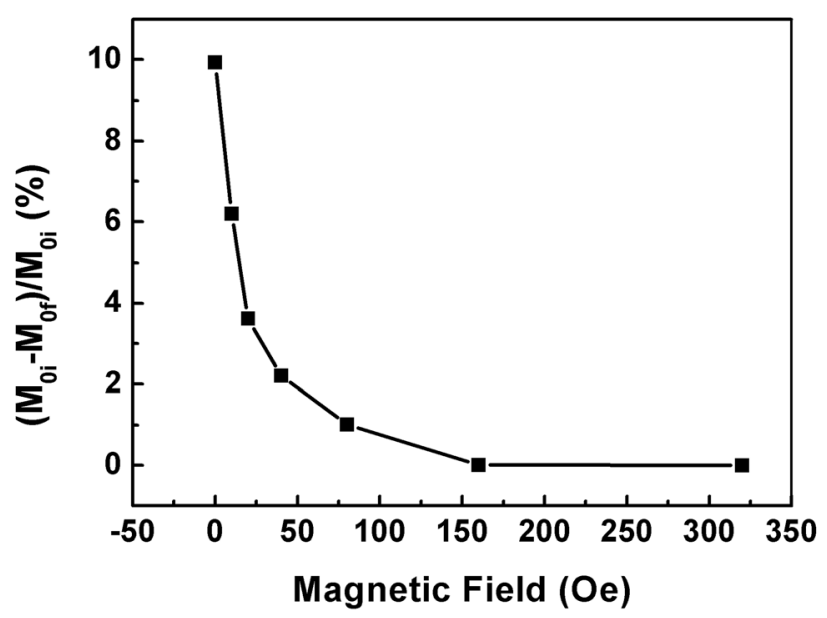

FIG. 9. The value of $\left(M_{O i}-M_{O f}\right) / M_{O i} \times 100 \%$ as a function of the bias magnetic field.

$15 \mathrm{kOe}$, and we recorded a new cycle on the sample. The difference $\left(M_{i 3}-M_{f 3}\right)$ observed in cycle 3 is the same as the one registered for cycle 1 , indicating that the initial magnetization is reversible. The same effect is observed whatever the cycle selected $\left(M_{i}\right.$ and $M_{f}$ points).

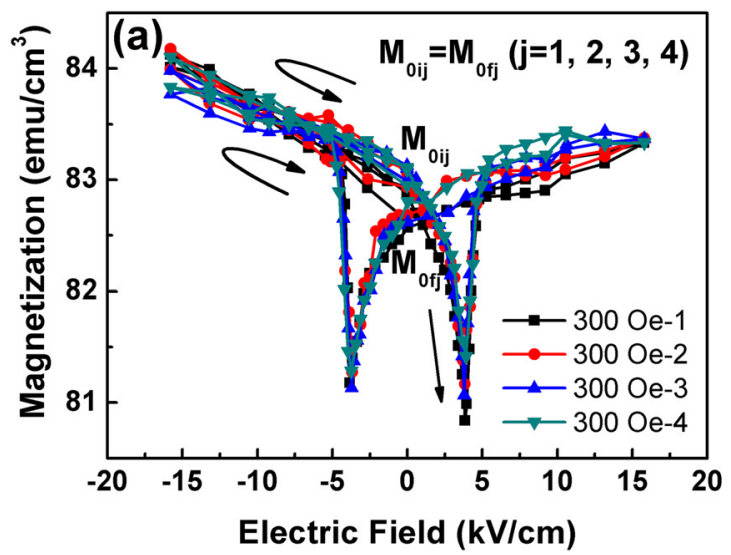

The converse coupling coefficients $(\alpha)$ can be obtained from the first derivative of the $M(E)$ curves $^{27}$ according to the equation $\alpha=\mu_{0} \delta M / \delta E\left(\mu_{0}=4 \pi \times 10^{-7} \mathrm{H} / \mathrm{m}\right)$. The maximum value of $\alpha$ found near $E c$ of the ceramic in each $\alpha(E)$ curve obtained as a function of the bias $\mathrm{H}$ field is collected to plot Fig. 12. The maximum value, $11 \times 10^{-8} \mathrm{~s} / \mathrm{m}$, is obtained at $H$ $=H c$. This value is surely not the maximum achievable for artificial multiferroic heterostructures because obviously the YIG is not the best choice for the magnetic material. It is quite difficult to give the highest value of the CME coupling reachable because it closely depends on the heterostructure arrangement (i.e., the substrate, the magnetic material...). Even so, the maximum value obtained is comparable to the one found for LSMO/PMN-PT (001). ${ }^{28}$ Moreover, this choice was not made particularly for the CME coupling performance but mainly on the possibility to integrate a composite material which owns good properties for future applications at high frequencies. YIG has been found to be a good compromise for this purpose.

Our main future application will be the fabrication of tunable inductances, and so, it is important to quantify the YIG film magnetization variation which will induce in turn the susceptibility (permeability) variations. In addition, the selection of PMN-PZT which can be obtained by sputtering lets us envisage positively a transfer towards a full thin film heterostructure. Actually, the final objective is the fabrication of artificial multiferroic heterostructures compatible with MMIC circuits. In this framework, it is essential to evaluate the potential of these kinds of heterostructures in terms of the susceptibility level achievable. To that end, Figure 13(a) presents the results obtained when measuring the variation of the permeability (susceptibility) according to the electric field and to the magnetic state of the film. We observe that between $0 \mathrm{kV} /$ $\mathrm{cm}$ and $-4 \mathrm{kV} / \mathrm{cm}$ applied to the piezoelectric ceramic, the relative susceptibility $\left(\chi_{\max }-\chi_{\min }\right) / \chi_{\max }$ exhibits a maximum of $16.8 \%$ for an $H$ value near to $H c$.

\section{CONCLUSIONS}

In summary, the electric field influence on the magnetic properties has been studied in a heterostructure composed of

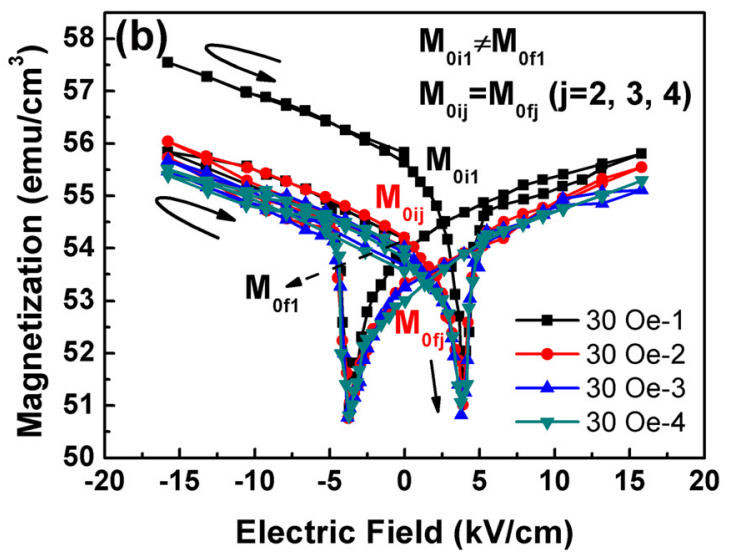

FIG. 10. The magnetization vs. electric field curves under bias magnetic fields of 300 Oe (a) and 30 Oe (b), respectively. Each curve is repeated 4 times, and there is no resaturation between the cycles. $M_{O i j}$ and $M_{O f j}(j=1,2,3$, and 4$)$ represent the initial and final magnetization states at $0 \mathrm{kV} / \mathrm{cm}$ in the $j$ cycle. All the curves can be traced by the arrows. 


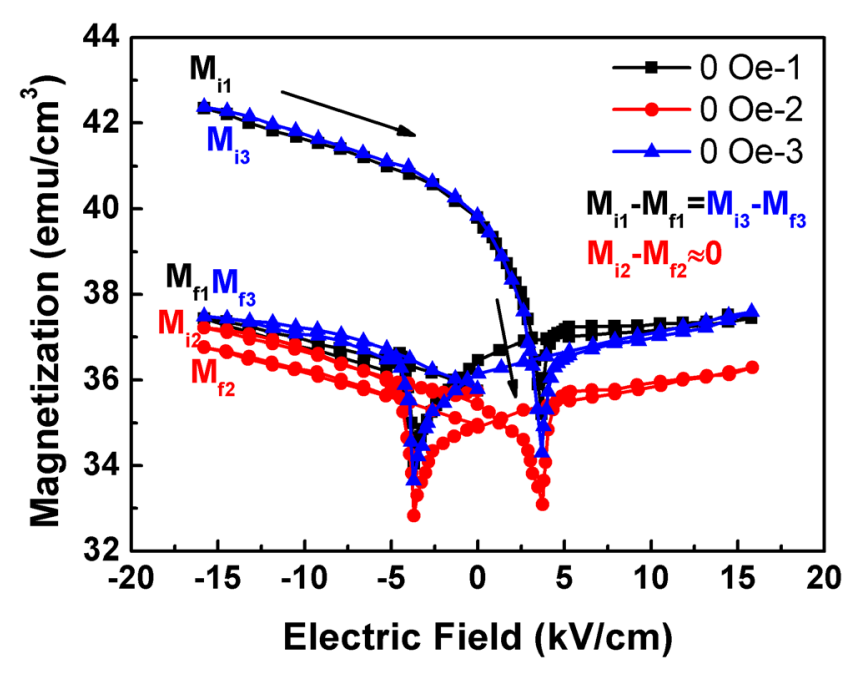

FIG. 11. Three different magnetization vs. electric field curves under 0 Oe. Before the first and third cycles, the sample is magnetically saturated, but no resaturation is applied to the film before the second cycle. $M_{i j}$ and $M_{f j}(j=1$, 2 , and 3) represent the initial and the final magnetization at $-15 \mathrm{kV} / \mathrm{cm}$ in the $j$ cycle. All the curves can be traced by the arrows.

a $200 \mathrm{~nm}$ thick YIG film deposited on a $0.5 \mathrm{~mm}$ thick PMNPZT ceramic with Pt on both sides, when the YIG film is in different magnetic states. It is shown that every magnetization-electric field curve is in good agreement with the ceramic strain evolution and the piezoelectric strain due to the DC voltage applied to the PMN-PZT ceramic controls the YIG magnetic properties. Moreover, it is always at $H c$ that the effect of the electric field is the maximum. Specifically, when the film is strongly magnetized $(H>H c)$, little variation due to the strain is observed. The amplitude is maximum at the coercive magnetic field. Another phenomenon, which modifies the film magnetization, also appears when the film is weakly magnetized $(H \leq H c)$. The straininduced changes in magnetization $\Delta M / M_{O i}$ and the $\mathrm{CME}$ coupling coefficient reach their maximum at the coercive magnetic field of the YIG film $\left(7.0 \%\right.$ and $10.9 \times 10^{-8} \mathrm{~s} / \mathrm{m}$, respectively), and even in the absence of an external magnetic field, there is still a large CME coupling coefficient

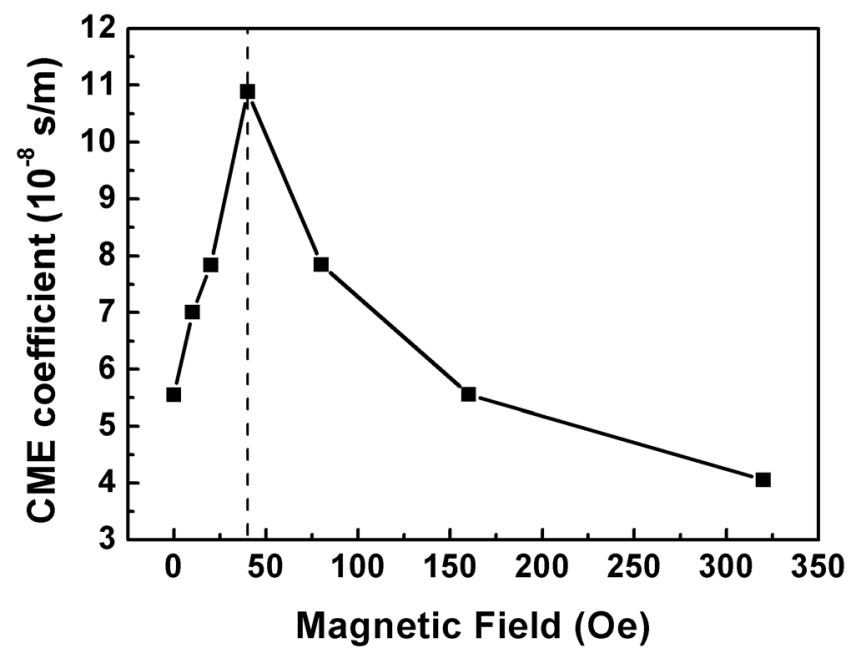

FIG. 12. The maximum CME coefficients in $\alpha(E)$ curves as a function of the bias magnetic field.

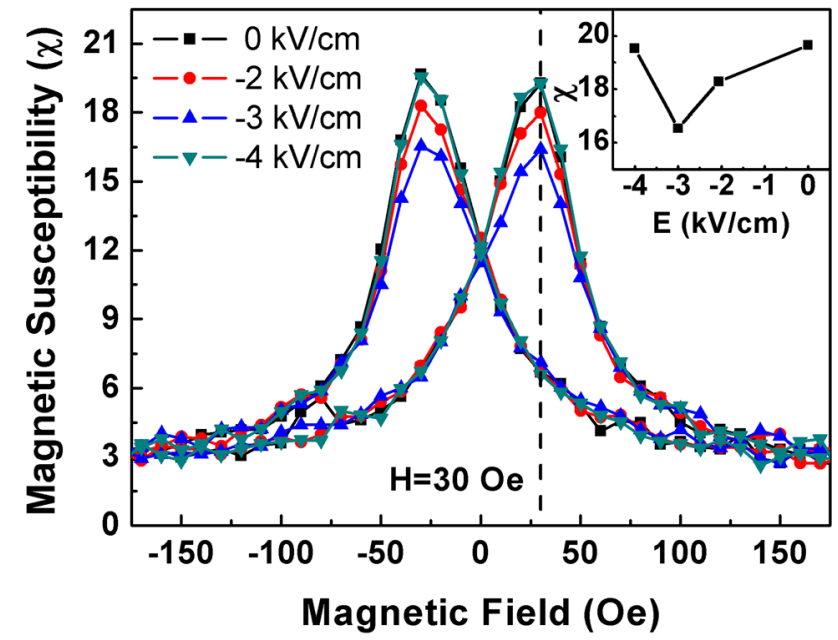

FIG. 13. The magnetic susceptibility $(\chi)$ vs. magnetic field curves originated from the first derivative of the corresponding $M(H)$ loops under different external electric fields $(0,-2,-3$, and $-4 \mathrm{kV} / \mathrm{cm})$, and the inset shows the maximum $\chi$ as a function of the external electric fields.

$\left(5.6 \times 10^{-8} \mathrm{~s} / \mathrm{m}\right)$. Experimental results also demonstrate that the strain produced by the converse piezoelectric effect of ceramics can not only control the magnetic properties of the magnetic film but also induce a loss of a part of the magnetization. The amount of the demagnetization is in one-to-one correspondence with the bias magnetic field and increases as the magnetic field decreases. When the YIG film undergoes the effect of strain, it does not return to its initial magnetic state, i.e., $M_{i}$ and $M_{f}$ states are different, with $M_{f}<M_{i}$. The greatest value for $M_{i}-M_{f}$ is on the order of $5 \mathrm{emu} / \mathrm{cm}^{3}$. It is certainly the consequence that under low magnetic fields, the magnetic moments are easily switched and the induced strain modifies some magnetic moment distribution. Under high magnetic fields, where the magnetic moments are strongly aligned, no difference has been observed $\left(M_{i}=M_{f}\right)$. Finally, we have demonstrated that it is possible to restore the initial magnetic state by applying a magnetic field $(1.5 \mathrm{~T})$ to the film to generate a reorientation of the moments as before.

Although the objective of this study is not to obtain the best CME coefficient, this latter was estimated. A maximum value of $11 \times 10^{-8} \mathrm{~s} / \mathrm{m}$ at the coercive magnetic field (40 Oe) and under a low applied electric field $(4.1 \mathrm{kV} / \mathrm{cm})$ has been found. The sharp deformation of about $2.5 \%$ of 0.25 PMN-0.75 PZT is at the origin of this excellent value of the coefficient CME.

Some parameters are needed to be studied in more detail to improve the strength of the CME. In particular, an efficient control and the manipulation of ferromagnetism by the induced strain in our structure necessitate perfect knowledge of the interactions across heterointerfaces and the YIG film thickness. Domains, domain walls and their mobility, and defects also play a critical role in the coupling phenomena.

The results reported in this study open up possibilities for device applications based on $E$-field controlled magnetism in heterostructures. With the choice of YIG, we know that the performance in terms of CME coefficient was limited, but the objective is to develop specific applications at 
microwave frequency where YIG materials have shown a real potential.

\section{ACKNOWLEDGMENTS}

This work was supported by the International Partnership Program of Chinese Academy of Sciences (Grant No. GJHZ1821), the National Natural Science Foundation of China (U153010140), and the Chinese Academy of Sciences President's International Fellowship Initiative (Grant No. 2017VEA0002).

${ }^{1}$ M. Fiebig, J. Phys. D: Appl. Phys. 38, R123 (2005).

${ }^{2}$ W. Eerenstein, N. D. Mathur, and J. F. Scott, Nature 442, 759 (2006).

${ }^{3}$ R. Ramesh and N. A. Spaldin, Nat. Mater. 6, 21 (2007)

${ }^{4}$ H. Ahmad, J. Atulasimha, and S. Bandyopadhyay, Nanotechnology 26, 401001 (2015).

${ }^{5}$ J. M. Rondinelli, M. Stengel, and N. A. Spaldin, Nat. Nanotechnol. 3, 46 (2008).

${ }^{6}$ Y. H. Chu, L. W. Martin, M. B. Holcomb, M. Gajek, S. J. Han, Q. He, N. Balke, C. H. Yang, D. Lee, W. Hu et al., Nat. Mater. 7, 478 (2008).

${ }^{7}$ Z. Y. Zhou, M. Trassin, Y. Gao, D. N. Qiu, K. Ashraf, T. X. Nan, X. Yang, S. R. Bowden, D. T. Pierce, M. D. Stiles et al., Nat. Commun. 6, $6082(2015)$.

${ }^{8}$ A. Klimov, N. Tiercelin, Y. Dusch, S. Giordano, T. Mathurin, P. Pernod, V. Preobrazhensky, A. Churbanov, and S. Nikitov, Appl. Phys. Lett. 110, 222401 (2017).

${ }^{9}$ T. Nan, M. Liu, W. Ren, Z. G. Ye, and N. X. Sun, Sci. Rep. 4, 5931 (2014).

${ }^{10}$ Y. Wang, J. Hu, Y. Lin, and C. W. Nan, NPG Asia Mater. 2, 61 (2010).

${ }^{11}$ M. I. Bichurin, I. A. Kornev, V. M. Petrov, A. S. Tatarenko, Y. V. Kiliba, and G. Srinivasan, Phys. Rev. B 64, 094409 (2001).
${ }^{12}$ H. Palneedi, V. Annapureddy, S. Priya, and J. Ryu, Actuators 5, 9 (2016)

${ }^{13}$ N. Jedrecy, H. J. von Bardeleben, V. Badjeck, D. Demaille, D. Stanescu, H. Magnan, and A. Barbier, Phys. Rev. B 88, 121409 (2013).

${ }^{14}$ M. Liu, Z. Zhou, T. Nan, B. M. Howe, G. J. Brown, and N. X. Sun, Adv. Mater. 25, 1435 (2013).

${ }^{15}$ T. Nozaki, Y. Shiota, S. Miwa, S. Murakami, F. Bonell, S. Ishibashi, H. Kubota, K. Yakushiji, T. Saruya, A. Fukushima et al., Nat. Phys. 8, 491 (2012).

${ }^{16}$ M. Liu and N. X. Sun, Philos. Trans. R. Soc. A 372, 20120439 (2014).

${ }^{17}$ L. W. Martin, S. P. Crane, Y. H. Chu, M. B. Holcomb, M. Gajek, M. Huijben, C. H. Yang, N. Balke, and R. Ramesh, J. Phys.: Condens. Matter 20, 434220 (2008).

${ }^{18}$ I. V. Zavislyak, M. A. Popov, G. Sreenivasulu, and G. Srinivasan, Appl. Phys. Lett. 102, 222407 (2013).

${ }^{19}$ G. Liu, X. Cui, and S. Dong, J. Appl. Phys. 108, 094106 (2010).

${ }^{20}$ J. Lou, D. Reed, M. Liu, and N. X. Sun, Appl. Phys. Lett. 94, 112508 (2009).

${ }^{21}$ Y. K. Fetisov and G. Srinivasan, Appl. Phys. Lett. 88, 143503 (2006).

${ }^{22}$ J. D. Adam, L. E. Davis, G. F. Dionne, E. F. Schloemann, and S. N. Stitzer, IEEE Trans. Microwave Theory Tech. 50, 721 (2002).

${ }^{23}$ C. S. Tsai and J. Su, Appl. Phys. Lett. 74, 2079 (1999).

${ }^{24}$ L. Wang, R. H. Liang, C. L. Mao, G. Du, G. S. Wang, and X. L. Dong, Ceram. Int. 39(7), 8571 (2013).

${ }^{25}$ S. Priya, R. Islam, S. X. Dong, and D. Viehland, J. Electroceram. 19, 149 (2007).

${ }^{26}$ J. Y. Lian, Y. Chen, Z. Liu, M. K. Zhu, G. S. Wang, W. B. Zhang, and X. L. Dong, Ceram. Int. 43(10), 7477 (2017).

${ }^{27}$ J. Y. Lian, F. Ponchel, N. Tiercelin, Y. Chen, D. Rémiens, T. Lasri, G. S. Wang, P. Pernod, W. B. Zhang, and X. L. Dong, Appl. Phys. Lett. 112, 162904 (2018).

${ }^{28}$ C. Thiele, K. Dörr, O. Bilani, J. Rödel, and L. Schultz, Phys. Rev. B 75, 054408 (2007). 